Pacific

Journal of

Mathematics

\title{
ON A SPECTRAL THEOREM IN PARAORTHOGONALITY THEORY
}

Kenier CASTillo, Ruymán CRUZ-BARRoso ANd Francisco Perdomo-Pío 


\title{
ON A SPECTRAL THEOREM IN PARAORTHOGONALITY THEORY
}

\author{
Kenier Castillo, Ruymán Cruz-Barroso \\ AND FranCISCO PERDOMO-Pío
}

\begin{abstract}
Motivated by the works of Delsarte and Genin (1988, 1991), who studied paraorthogonal polynomials associated with positive definite Hermitian linear functionals and their corresponding recurrence relations, we provide paraorthogonality theory, in the context of quasidefinite Hermitian linear functionals, with a recurrence relation and the analogous result to the classical Favard's theorem or spectral theorem. As an application of our results, we prove that for any two monic polynomials whose zeros are simple and strictly interlacing on the unit circle, with the possible exception of one of them which could be common, there exists a sequence of paraorthogonal polynomials such that these polynomials belong to it. Furthermore, an application to the computation of Szegó quadrature formulas is also discussed.
\end{abstract}

\section{Introduction}

The paraorthogonal polynomials on the unit circle (POPUC), in the context of quasidefinite (or regular) moment linear functionals, were introduced for the first time by Jones, Njåstad and Thron in their excellent survey paper [Jones et al. 1989]. The main objective of the authors was to construct quadrature formulas for the approximation of an integral with respect to a measure whose support is contained in the unit circle, analogous to the generalized Gaussian rules and, as a consequence, solve the trigonometric moment problem [Geronimus 1946]. In this respect, nodes on the unit circle, positive weights and maximal domain of validity are required. As a result, the so-called Szegó quadrature (SQ) formulas are introduced and characterized: their nodes are zeros of a special class of POPUC, known as invariant (or self-inverse). But moreover, [Jones et al. 1989] served to demand a deeper study of the properties of this new family of polynomials since, contrary to orthogonal polynomials on the unit circle (OPUC), the invariant POPUC with respect to a measure supported on the unit circle have simple zeros on the unit circle with many additional

MSC2010: 42C05, 30C15, 26C10.

Keywords: paraorthogonal polynomials, quasidefinite Hermitian linear functionals, spectral theorem,

Geronimus-Wendroff theorem, Szegó quadrature formulas. 
properties [Cantero et al. 2002; Golinskii 2002; Simon 2005a; 2005b; 2007; 2011; Wong 2007]. These polynomials play in the unit circle the same role as orthogonal polynomials on the real line (OPRL) from the perspective of quadrature formulas.

The earliest reference to invariant POPUC is due to Geronimus [1946, Theorem III]. However, in [Jones et al. 1989] they are defined in a more general setting from their orthogonality conditions and characterized in terms of the corresponding OPUC. The counterpart to the deficiency in the orthogonality conditions for POPUC, which are not orthogonal to the constants, is the fact that for a given measure and a fixed $n$, the POPUC of degree $n$ is not unique, and basically depends on one unimodular free parameter. Equivalently, in quadrature terminology, we have a one-parameter family of $n$-point SQ formulas, exact in a subspace of Laurent polynomials of dimension $2 n-1$, instead of $2 n$; see, e.g., [Cruz-Barroso et al. 2007; Peherstorfer 2011].

Beyond their essential role in the development of quadrature formulas, the theory of invariant POPUC is significantly enriched from both theoretical and practical points of view. The uses of their zeros instead of zeros of OPUC in frequency analysis problems [Daruis et al. 2003] and their appearance as the minimizer of the isometric Arnoldi minimization problem [Helsen et al. 2005] represent some of their best applications. On the other hand, the works of Cantero, Moral and Velázquez [Cantero et al. 2002], Golinskii [2002], Simon [2007], and Wong [2007] are essential to understand the behavior of the zeros of POPUC. Recently in [Simanek 2015], it was also proved that the zeros of invariant POPUC designate the location of a set of particles that are in electrostatic equilibrium with respect to a particular external field. Furthermore, after their formal introduction, POPUC were defined in the more general context of orthogonal rational functions [Bultheel et al. 1999, Chapter 5].

One of the main algebraic properties in the study of orthogonal polynomials has not been established yet for general POPUC in the context of quasidefinite linear functionals: a recurrence relation and its corresponding Favard's theorem or spectral theorem. And this is precisely the starting point of this work, even though for the positive definite case it is very well known that POPUC satisfy a three-term recurrence, which is the key for the tridiagonal approach developed by Delsarte and Genin [1988; 1991a; 1991b] to solve the standard linear prediction problem. Similar results can be also found in [Castillo et al. 2014] where the corresponding OPUC and the nontrivial probability measure supported on the unit circle are deduced. The reader whose interest concerns particularly the applications of POPUC to digital signal processing can find a survey in [Delsarte and Genin 1990]. We recall that in [Delsarte and Genin 1988; 1991a; 1991b], the authors considered POPUC associated with positive definite moment linear functionals [Delsarte and Genin 1988, (4.13)] and, particularly, in [loc. cit.] they say that presumably the quasidefinite case can be traced with the help of the theory of pseudo-Carathéodory functions. Motivated by this last observation, in the present work, we study some properties of POPUC for the quasidefinite 
case using only standard techniques from the theory of OPRL and OPUC. We focus our attention on the analogs of the spectral theorem and the Geronimus-Wendroff theorem for POPUC, expecting them to be as useful as these results in the theory of OPRL and OPUC. The importance of the previous results for OPRL and OPUC is summarized in the survey [Marcellán and Álvarez-Nodarse 2001].

In Section 3, we prove that three consecutive POPUC are connected by a simple relation which we can derive in a straightforward way. Moreover, the spectral theorem is also proved. In Section 4, we present an example of the applicability of the spectral theorem by proving the Geronimus-Wendroff theorem for POPUC. Furthermore, an application to the computation of SQ formulas is considered.

In the next section, we fix the notation used in this work and present some preliminaries, which will help to make our original results self-contained and accessible to the reader not familiar with the theory of OPUC and POPUC.

\section{Orthogonality and paraorthogonality}

We denote by $\Lambda:=\mathbb{C}\left[z, z^{-1}\right]$ the complex vector space of Laurent polynomials in the variable $z$. Associated with every pair of integer numbers $(p, q)$, with $p \leq q$, we define the vector subspace $\Lambda_{p, q}$ of Laurent polynomials of the form

$$
\sum_{n=p}^{q} \varsigma_{n} z^{n}, \quad \varsigma_{n} \in \mathbb{C} .
$$

The vector subspace of complex polynomials will be denoted by $\mathbb{P}:=\mathbb{C}[z]$ and we write $\mathbb{P}_{q} \equiv \Lambda_{0, q}$ for the vector subspace of polynomials of degree (at most) $q$, while $\mathbb{P}_{-1} \equiv\{0\}$ is the trivial subspace.

Let us introduce the moment linear functional $\mu$ on $\Lambda$ such that

$$
c_{n}:=\mu\left(z^{n}\right)=\overline{\mu\left(z^{-n}\right)}=: \bar{c}_{-n}, \quad n \geq 0,
$$

i.e., $\mu$ is an Hermitian linear functional. The complex numbers $\left\{c_{n}\right\}_{n=-\infty}^{\infty}$ are called the moments associated with $\mu$. In terms of $\mu$, we consider a sesquilinear functional $\langle\cdot, \cdot\rangle$ on $\Lambda \times \Lambda$ defined by

$$
\langle f, g\rangle:=\mu\left(f(z) \bar{g}\left(z^{-1}\right)\right), \quad f, g \in \Lambda .
$$

The Gram matrix associated with the inner product $\langle\cdot, \cdot\rangle$ in terms of $1, z, z^{2}, \ldots$ is the Toeplitz matrix

$$
\boldsymbol{T}=\left[\left\langle z^{l}, z^{j}\right\rangle\right]_{l, j \geq 0}=\left[\begin{array}{ccccc}
c_{0} & c_{1} & \cdots & c_{n} & \cdots \\
c_{-1} & c_{0} & \cdots & c_{n-1} & \cdots \\
\vdots & \vdots & \ddots & \vdots & \\
c_{-n} & c_{-n+1} & \cdots & c_{0} & \cdots \\
\vdots & \vdots & & \vdots & \ddots
\end{array}\right] .
$$


Denote by $\boldsymbol{T}_{n}$ the $(n+1) \times(n+1)$ principal leading submatrix of $\boldsymbol{T}$. If $\operatorname{det}\left(\boldsymbol{T}_{n}\right) \neq 0$ for every $n \geq 0$, then $\mu$ is said to be quasidefinite and the existence of a sequence of monic polynomials, orthogonal with respect to $\mu$, is guaranteed. On the other hand, by the Carathéodory-Toeplitz theorem [Simon 2005a, Section 1.3], if $\operatorname{det}\left(\boldsymbol{T}_{n}\right)>0$ for every $n \geq 0$, then (2-1) are the moments of a nontrivial (i.e., with infinitely many points of increase) probability measure $d \sigma$ supported on the unit circle $\partial \mathbb{D}$, that is, the boundary of the open unit disk $\mathbb{D}:=\{z \in \mathbb{C} ;|z|<1\}$ parametrized by $z=e^{i \theta}, \theta \in[0,2 \pi)$, and the converse is also true. In mathematical terms, $\mu$ has the integral representation

$$
\mu(f)=\int f d \sigma, \quad f \in \Lambda .
$$

In this case, $\mu$ is called positive definite.

The application of the Gram-Schmidt process to $1, z, z^{2}, \ldots$ (a linearly independent system in the Hilbert space $L^{2}(\partial \mathbb{D}, d \sigma)$ with the norm induced by our inner product) yields the sequence of monic polynomials, $\left\{\Phi_{n}\right\}_{n \geq 0}$, orthogonal with respect to $d \sigma$ (or equivalently with respect to $\mu$ ) called the sequence of OPUC (see [Simon 2005a; 2005b; 2011] for a recent account of the theory). In other words, there exists a unique sequence of monic polynomials such that

$$
\left\langle\Phi_{n}, z^{m}\right\rangle=\int \Phi_{n}(z) z^{-m} d \sigma(z)=\kappa_{n} \delta_{n, m}, \quad \kappa_{n}>0, \quad 0 \leq m \leq n,
$$

with $\Phi_{n} \in \mathbb{P}_{n} \backslash \mathbb{P}_{n-1}$ and $\delta_{n, m}$ the Kronecker delta symbol. We recall that the solution of the trigonometric moment problem is always unique [Geronimus 1946; Jones et al. 1989]. The associated orthonormal polynomials are given by

$$
\varphi_{n}=\kappa_{n} \Phi_{n}, \quad \kappa_{n}:=\prod_{j=0}^{n-1} \rho_{j}^{-1}, \quad \rho_{j}:=\left(1-\left|\Phi_{j+1}(0)\right|^{2}\right)^{1 / 2} .
$$

The monic OPUC satisfy the following recurrence relation (Szegő's recurrence):

$$
\left[\begin{array}{l}
\Phi_{n+1}(z) \\
\Phi_{n+1}^{*}(z)
\end{array}\right]=\left[\begin{array}{cc}
z & -\bar{\alpha}_{n} \\
-\alpha_{n} z & 1
\end{array}\right]\left[\begin{array}{l}
\Phi_{n}(z) \\
\Phi_{n}^{*}(z)
\end{array}\right]
$$

with initial condition $\Phi_{0}: \equiv 1$. The numbers $\left\{\alpha_{n}\right\}_{n \geq 0} \in \mathbb{D}^{\infty}$ are known as Verblunsky coefficients and, as usual, if $f \in \mathbb{P}_{n} \backslash \mathbb{P}_{n-1}$, then $f^{*}$ denotes its reversed polynomial, defined by $f^{*}(z):=z^{n} \overline{f(1 / \bar{z})}$. By Szegô's recurrence, we get $\alpha_{n}=-\overline{\Phi_{n+1}(0)}$ since $\Phi_{n}^{*}(0)=1$; thus, we set $\alpha_{-1}: \equiv-1$. We recall that for the quasidefinite case $\left\{\alpha_{n}\right\}_{n \geq 0} \notin \partial \mathbb{D}^{\infty}$. 
The orthogonality conditions (2-2) can be weakened adequately in order to overcome the apparent difference between OPRL and OPUC [Simon 2011, Theorem 1.2.6 and Theorem 2.14.2]. In this way, the corresponding polynomials will be the POPUC introduced by Jones, Njåstad and Thron [Jones et al. 1989].

Definition 2.1. A sequence of polynomials $\left\{\Phi_{n}\left(\cdot, \tau_{n}\right)\right\}_{n \geq 0}$ is said to be a sequence of POPUC if

$$
\begin{gathered}
\left\langle\Phi_{n}\left(\cdot, \tau_{n}\right), 1\right\rangle \neq 0, \\
\left\langle\Phi_{n}\left(z, \tau_{n}\right), z^{m}\right\rangle=0, \quad 1 \leq m \leq n-1, \quad\left\langle\Phi_{n}\left(z, \tau_{n}\right), z^{n}\right\rangle \neq 0 .
\end{gathered}
$$

In general, we follow the notation from standard literature, as in [Simon 2005a; $2005 \mathrm{~b} ; 2011]$. The presence of the parameter $\tau_{n}$ in Definition 2.1 will be fully clarified in (2-6); see further. It is worth pointing out that in the applications (see among others [Jones et al. 1989; Peherstorfer 2011]), it is useful to have a POPUC such that the distribution of its zeros behaves as in the case of OPRL. This allows us to introduce the concept of invariance.

Definition 2.2. A sequence of polynomials $\left\{f_{n}\right\}_{n \geq 0}$ is said to be invariant if there exists $\chi_{n} \in \partial \mathbb{D}$ such that $f_{n}^{*}=\chi_{n} f_{n}$.

By [Jones et al. 1989, Theorem 6.1(B)], if the polynomials $\left\{\Phi_{n}\left(\cdot, \tau_{n}\right)\right\}_{n \geq 1}$ are (monic) invariant POPUC, then

$$
\Phi_{n}\left(z, \tau_{n}\right)=z \Phi_{n-1}(z)-\bar{\tau}_{n} \Phi_{n-1}^{*}(z),
$$

with $\tau_{n}=-\chi_{n} \in \partial \mathbb{D}$. Clearly, the converse is also true. Based on the previous assertion, $\Phi_{n}\left(\cdot, \tau_{n}\right)$ is completely determined by the parameter $\tau_{n}$ and the first $n-1$ Verblunsky coefficients associated with the corresponding sequence of OPUC.

For numbers on $\partial \mathbb{D}$, we can define a cyclic order in terms of their arguments [Simon 2007]. An ordered set of points $\left(z_{1}, \ldots, z_{n}\right) \in \partial \mathbb{D}^{n}$ is called cyclicly ordered if $\left(z_{j}, z_{j+1}\right)_{j=1}^{n}$ and $\left(z_{n}, z_{1}\right)$ contain no other $z_{j}$. Two cyclicly ordered sets of points on $\partial \mathbb{D},\left(z_{1}, \ldots, z_{n}\right)$ and $\left(\zeta_{1}, \ldots, \zeta_{m}\right)$, are said to strictly interlace if after a cyclic permutation of the $\zeta_{j}$, we have $\zeta_{j} \in\left(z_{j}, z_{j+1}\right), j=1, \ldots, m$, and $\zeta_{n} \in\left(z_{n}, z_{1}\right)$ if $n=m$. This definition can be naturally extended to two cyclicly ordered sets of zeros with different numbers of elements. We recall here that the zeros of two consecutive invariant POPUC, $\Phi_{n+1}\left(\cdot, \tau_{n+1}\right)$ and $\Phi_{n}\left(\cdot, \tau_{n}\right)$, associated with the measure $d \sigma$ (positive definite case) have at most one zero in common, namely $\zeta$. In other words, one of two following possibilities holds: the zeros of $\Phi_{n+1}\left(z, \tau_{n+1}\right) /(z-\zeta)$ and $\Phi_{n}\left(\cdot, \tau_{n}\right)$, or the zeros of $\Phi_{n+1}\left(\cdot, \tau_{n+1}\right)$ and $\Phi_{n}\left(\cdot, \tau_{n}\right)$, strictly interlace on $\partial \mathbb{D}$ (see [Simon 2007] and the references given there). We must once again urge the reader to consult the monographs [Simon 2005a; 2005b; 2011] where all the previous results can be found. 


\section{Recurrence relation and spectral theorem}

It is very well known that the OPRL (and also the OPUC for nonzero Verblunsky coefficients) satisfy a linear recurrence relation [Chihara 1978; Szegô 1975] which plays a crucial role in the subsequent behavior of their theory. Such a recurrence relation does not hold for POPUC, but we can obtain a similar recurrence formula. To do this, we follow an analogous procedure to one pointed out by Atkinson [1964] and recovered by Simon [2005a, Theorem 1.5.2] to obtain Szegó's recurrence.

Theorem 3.1. Given a quasidefinite moment functional $\mu$, there always exist three consecutive monic POPUC such that

$$
\Phi_{n+1}\left(z, \tau_{n+1}\right)=\left(z+\beta_{n}\right) \Phi_{n}\left(z, \tau_{n}\right)-\gamma_{n} z \Phi_{n-1}\left(z, \tau_{n-1}\right),
$$

where $\beta_{n}, \gamma_{n} \in \mathbb{C} \backslash\{0\}$ are given by

$$
\gamma_{n}=\frac{\left\langle\Phi_{n}\left(\cdot, \tau_{n}\right), 1\right\rangle}{\left\langle\Phi_{n-1}\left(\cdot, \tau_{n-1}\right), 1\right\rangle}, \quad \beta_{n}=\gamma_{n} \frac{\left\langle\Phi_{n-1}\left(z, \tau_{n-1}\right), z^{n-1}\right\rangle}{\left\langle\Phi_{n}\left(z, \tau_{n}\right), z^{n}\right\rangle}
$$

Proof. Our proof starts with the observation that

$$
Q_{n+1}(z):=\left(z+\beta_{n}\right) \Phi_{n}\left(z, \tau_{n}\right)-\gamma_{n} z \Phi_{n-1}\left(z, \tau_{n-1}\right), \quad \beta_{n}, \gamma_{n} \in \mathbb{C},
$$

is a monic polynomial of degree $n+1$ which is orthogonal to $\operatorname{span}\left\{z^{2}, z^{3}, \ldots, z^{n-1}\right\}$. The important point to notice here is that for constants $\beta_{n}$ and $\gamma_{n}$ given as in (3-8), $Q_{n+1}(z)$ is orthogonal to $\operatorname{span}\left\{z, z^{2}, \ldots, z^{n}\right\}$, which proves the theorem.

In terms of the parameters $\left\{\tau_{n}\right\}_{n \geq 1}$, the previous theorem says that given two numbers $\tau_{n-1}$ and $\tau_{n}$, a third number $\tau_{n+1}$ can be found such that the corresponding POPUC satisfies (3-7). We are now interested in the expression of the recurrence coefficients (3-8) in terms of the parameters $\left\{\tau_{n}\right\}_{n \geq 1} \in \partial \mathbb{D}^{\infty}$ and the Verblunsky coefficients.

Corollary 3.2. With reference to the recurrence formula (3-7) for the invariant case, the following holds:

$$
\beta_{n}=\frac{\tau_{n}}{\tau_{n+1}} \in \partial \mathbb{D}, \quad \gamma_{n}=\frac{\tau_{n}-\alpha_{n-1}}{\tau_{n+1}-\alpha_{n-2}} \rho_{n-2}^{2} \in \mathbb{C} \backslash\{0\} .
$$

Proof. From (2-6), we get

$$
\Phi_{n}^{*}\left(\cdot, \tau_{n}\right)=-\tau_{n} \Phi_{n}\left(\cdot, \tau_{n}\right)
$$

which gives

$$
\tau_{n+1} \Phi_{n+1}\left(z, \tau_{n+1}\right)=\left(1+\bar{\beta}_{n} z\right) \tau_{n} \Phi_{n}\left(z, \tau_{n}\right)-\bar{\gamma}_{n} z \tau_{n-1} \Phi_{n-1}\left(z, \tau_{n-1}\right),
$$

when substituted in the reversed (3-7). The expression for $\beta_{n}$ follows from the above equation comparing the leading coefficients. 
By inverting the Szegó recurrence (2-4) [Simon 2005a, Theorem 1.5.4], it is easy to check that (2-6) in the monic case can be expressed by

$$
\Phi_{n}\left(z, \tau_{n}\right)=\frac{1}{1-\omega_{n} \alpha_{n-1}}\left(\Phi_{n}(z)+\omega_{n} \Phi_{n}^{*}(z)\right), \quad \omega_{n}:=\frac{\bar{\alpha}_{n-1}-\bar{\tau}_{n}}{1-\bar{\tau}_{n} \alpha_{n-1}} .
$$

Thus, $\gamma_{n}$ follows from here as a consequence of Theorem 3.1, (2-3), the paraorthogonality conditions for $\Phi_{n}\left(\cdot, \tau_{n}\right)$ and the orthogonality conditions for $\Phi_{n}$ and $\Phi_{n}^{*}$.

The following result will be useful in determining the relation between $\tau_{n-1}, \tau_{n}$, and $\tau_{n+1}$ for the invariant case.

Lemma 3.3. Let $\left\{\beta_{n}\right\}_{n \geq 0}$ be an arbitrary sequence on $\partial \mathbb{D}$ and let $\left\{\gamma_{n}\right\}_{n \geq 1}$ be an arbitrary sequence on $\mathbb{C} \backslash\{0\}$. Any sequence of polynomials $\left\{\Psi_{n}\right\}_{n \geq 0}$ defined by

$$
\Psi_{n+1}(z)=\left(z+\beta_{n}\right) \Psi_{n}(z)-\gamma_{n} z \Psi_{n-1}(z),
$$

with initial conditions $\Psi_{0}: \equiv 1$ and $\Psi_{1}(z):=z+\beta_{0}$, is a sequence of invariant polynomials if and only if the recurrence coefficients satisfy

$$
\frac{\gamma_{n}}{\bar{\gamma}_{n}}=\beta_{n-1} \beta_{n}
$$

Proof. As a direct consequence of Theorem 3.1, we get

$$
\Psi_{n}(z)=\operatorname{det}\left(J_{n}(z)\right), \quad n \geq 1,
$$

where the matrix $\boldsymbol{J}_{n}(z)$ is given by

$$
\boldsymbol{J}_{n}(z)=\left[\begin{array}{cccccc}
z+\beta_{0} & -\gamma_{1} & 0 & \cdots & 0 & 0 \\
-z & z+\beta_{1} & -\gamma_{2} & \cdots & 0 & 0 \\
0 & -z & z+\beta_{2} & \cdots & 0 & 0 \\
\vdots & \vdots & \vdots & \ddots & \vdots & \vdots \\
0 & 0 & 0 & \cdots & z+\beta_{n-1} & -\gamma_{n-1} \\
0 & 0 & 0 & \cdots & -z & z+\beta_{n-1}
\end{array}\right] .
$$

For a polynomial $f$ to be invariant, it is necessary and sufficient that $|f| \equiv\left|f^{*}\right|$. Let us define the matrices $\boldsymbol{D}_{n}:=\operatorname{diag}\left[\beta_{0}^{-1}, \beta_{1}^{-1}, \ldots, \beta_{n-1}^{-1}\right]$ and

$$
\boldsymbol{J}_{n}^{(0)}(z):=\left[\begin{array}{cccccc}
z+\beta_{0} & \beta_{0} & 0 & \cdots & 0 & 0 \\
-\bar{\gamma}_{1} \beta_{1} z & z+\beta_{1} & \beta_{1} & \cdots & 0 & 0 \\
0 & -\bar{\gamma}_{2} \beta_{2} z & z+\beta_{2} & \cdots & 0 & 0 \\
\vdots & \vdots & \vdots & \ddots & \vdots & \vdots \\
0 & 0 & 0 & \cdots & z+\beta_{n-2} & \beta_{n-2} \\
0 & 0 & 0 & \cdots & \bar{\gamma}_{n-1} \beta_{n-1} z & z+\beta_{n-1}
\end{array}\right] .
$$


Notice that $z \boldsymbol{J}_{n}^{H}(1 / \bar{z})=\boldsymbol{D}_{n} \boldsymbol{J}_{n}^{(0)}(z)$, where the ${ }^{H}$-operator denotes the conjugate transpose. Hence,

$$
\left|\operatorname{det}\left(\boldsymbol{J}_{n}(z)\right)\right|=\left|\operatorname{det}\left(\boldsymbol{D}_{n}\right)\right|\left|\operatorname{det}\left(\boldsymbol{J}_{n}^{(0)}(z)\right)\right|=\left|\operatorname{det}\left(z \boldsymbol{J}_{n}^{H}(1 / \bar{z})\right)\right|, \quad n \geq 1,
$$

holds, if and only if (3-11) holds.

Notice that under the hypothesis of Corollary 3.2, we get by Lemma 3.3 that

$$
\tau_{n+1}=\frac{\bar{\gamma}_{n}}{\gamma_{n}} \tau_{n-1}
$$

As a consequence, we can deduce the following (forward) recurrence relation for the paraorthogonality parameters,

$$
\tau_{n+1}=\frac{\tau_{n}-\alpha_{n-1}}{\bar{\tau}_{n}-\bar{\alpha}_{n-1}} \frac{\bar{\tau}_{n-1}-\bar{\alpha}_{n-2}}{\tau_{n-1}-\alpha_{n-2}} \tau_{n-1},
$$

which is equivalent to the (backward) recurrence

$$
\tau_{n-1}=\frac{\tau_{n+1} \alpha_{n-2}\left(\bar{\alpha}_{n-1}-\bar{\tau}_{n}\right)+\alpha_{n-1}-\tau_{n}}{\tau_{n+1}\left(\bar{\alpha}_{n-1}-\bar{\tau}_{n}\right)+\bar{\alpha}_{n-2}\left(\alpha_{n-1}-\tau_{n}\right)} .
$$

We next point out the important converse of Theorem 3.1. In agreement with classical literature, we refer to this result as Favard's theorem (see, among others, [Chihara 1978; Erdélyi et al. 1991; Favard 1935; Marcellán and Álvarez-Nodarse 2001; Szegó 1975]) or the spectral theorem [Ismail 2005], even though this result is previously contained in the works of Stieltjes [1895; 1894] and Stone [1932]. In the positive definite case, an analog of Favard's theorem for OPUC based on the construction of a sequence of absolutely continuous measures whose limit is the spectral measure is presented in [Erdélyi et al. 1991]. In this paper the authors follow a method used previously by Delsarte, Genin and Kamp [Delsarte et al. 1978] who consider the matrix-valued case. Some extensions to the quasidefinite case have been analyzed in [Marcellán and Álvarez-Nodarse 2001]. Our proof follows a standard scheme (constructive approach) which goes back at last to [Chihara 1978]; see also [Marcellán and Álvarez-Nodarse 2001].

Theorem 3.4 (spectral theorem). Let $\left\{\beta_{n}\right\}_{n \geq 0}$ be an arbitrary sequence on $\partial \mathbb{D}$ and let $\left\{\gamma_{n}\right\}_{n \geq 1}$ be an arbitrary sequence on $\mathbb{C} \backslash\{0\}$. Set $c_{0} \in \mathbb{R} \backslash\{0\}$ and let $\left\{\Psi_{n}\right\}_{n \geq 0}$ be a sequence of invariant polynomials satisfying a recurrence relation as (3-10), with $\Psi_{0}: \equiv 1$ and $\Psi_{1}(z):=z+\beta_{0}$. Then there exists a unique quasidefinite moment functional $\mu$ such that $\mu(1)=c_{0}$ and $\left\{\Psi_{n}\right\}_{n \geq 0}$ is the corresponding sequence of POPUC. Moreover, if $\left\{\Psi_{n}\right\}_{n \geq 0}$ is a sequence of polynomials with all its zeros on $\partial \mathbb{D}$, then there exists a unique measure $d \sigma$ such that $\int d \sigma=c_{0}>0$ and $\left\{\Psi_{n}\right\}_{n \geq 0}$ is the corresponding sequence of POPUC. 
Proof. We begin by constructing for $n \geq 2$ the moment linear functional $\mu^{(n)}$ on $\Lambda_{-(n-1), n-1}$ by

$$
c_{k}:=\mu^{(n)}\left(z^{k}\right)=\overline{\mu^{(n)}\left(z^{-k}\right)}=: \bar{c}_{-k}, \quad 0 \leq k \leq n-1,
$$

such that

$$
\mu^{(n)}\left(\Psi_{\ell}(z) z^{-1}\right)=0, \quad 2 \leq \ell \leq n .
$$

So we proceed by induction on $n$. Notice that for $n=2$,

$$
\mu^{(2)}\left(\Psi_{2}(z) z^{-1}\right)=c_{1}+\beta_{0} \beta_{1} c_{-1}+\left(\beta_{0}+\beta_{1}-\gamma_{1}\right) c_{0},
$$

which allows us to define $c_{1}=\bar{c}_{-1} \in \mathbb{C}$. More precisely, we let $c_{1}:=\left(1 / 2 \gamma_{1}-\beta_{0}\right) c_{0}$, which by Lemma 3.3 implies

$$
\mu^{(2)}\left(\Psi_{2}(z) z^{-1}\right)=\left(1 / 2 \gamma_{1}+1 / 2 \bar{\gamma}_{1} \beta_{0} \beta_{1}-\gamma_{1}\right) c_{0}=0 .
$$

In order to prove (3-15), write

$$
\Psi_{n}(z)=z^{n}-\sum_{k=0}^{n-1} a_{n, k} \Psi_{k}(z),
$$

where $\left\{a_{n, k}\right\}_{k=0}^{n-1}$ is uniquely determined. Let us now define $\mu^{(n)}$ on $\Lambda_{-(n-1), n-1}$ as an extension of $\mu^{(n-1)}$ such that $c_{n-1}:=\left(a_{n, 0}+a_{n, 1} \beta_{0}\right) c_{-1}+a_{n, 1} c_{0}$. In other words, we assume that for some $n \geq 3, c_{-(n-2)}, \ldots, c_{n-2}$ have been determined such that $\mu^{(n-1)}$ defined on $\Lambda_{-(n-2), n-2}$ satisfies

$$
c_{m}=\bar{c}_{-m}, \quad 0 \leq m \leq n-2,
$$

and

$$
\mu^{(n-1)}\left(\Psi_{\ell}(z) z^{-1}\right)=0, \quad 2 \leq \ell \leq n-1 .
$$

Hence, by our assumption,

$$
\mu^{(n)}\left(\Psi_{n}(z) z^{-1}\right)=0 .
$$

This completes the induction. Therefore, it follows that $\mu$ defined on $\Lambda$ by (2-1) is an extension of $\mu^{(n)}$ defined on $\Lambda_{-n, n}$, and consequently

$$
\mu\left(\Psi_{n}(z) z^{-1}\right)=0, \quad n \geq 2 .
$$

According to the paraorthogonality conditions (2-5), it remains to check that

$$
\left\langle\Psi_{n}, 1\right\rangle \neq 0, \quad\left\langle\Psi_{n}(z), z^{q}\right\rangle=0, \quad 2 \leq q \leq n-1, \quad\left\langle\Psi_{n}(z), z^{n}\right\rangle \neq 0 .
$$


From (3-7) and (3-16), we obtain

$$
\begin{aligned}
\left\langle\Psi_{n}, 1\right\rangle & =\gamma_{n}\left\langle\Psi_{n-1}, 1\right\rangle \\
& =c_{0} \prod_{k=1}^{n} \gamma_{k}=: b_{0} \neq 0 .
\end{aligned}
$$

On the other hand, from (3-16) and the Hermitian character of the functional,

$$
\left\langle\Psi_{n}(z), z\right\rangle=\left\langle\Psi_{n}^{*}(z), z^{n-1}\right\rangle=0,
$$

which by the invariant hypothesis implies

$$
\left\langle\Psi_{n}(z), z^{n-1}\right\rangle=0 .
$$

Now, we define an appropriate statement $\left(I_{r}\right)$ by

$$
\left\langle\Psi_{n}(z), z^{q}\right\rangle=0, \quad 1 \leq q \leq r, \quad n \geq r+1,
$$

and prove by induction that the statement is valid for all $r$. Obviously, $\left(I_{1}\right)$ is (3-16). Assuming $\left(I_{r}\right)$ holds for some $r \geq 2$, we will prove $\left(I_{r+1}\right)$, that is,

$$
\left\langle\Psi_{n}(z), z^{r+1}\right\rangle=0, \quad n \geq r+2 .
$$

Since $\left(I_{r}\right)$ holds, (3-7) yields

$$
\left\langle\Psi_{n+1}(z), z^{r+1}\right\rangle=\beta_{n}\left\langle\Psi_{n}(z), z^{r+1}\right\rangle .
$$

Taking into account that (3-17) holds,

$$
\left\langle\Psi_{r+2}(z), z^{r+1}\right\rangle=0,
$$

which yields $\left\langle\Psi_{r+3}(z), z^{r+1}\right\rangle=0$. Continuing in this manner, we conclude that $\left(I_{r+1}\right)$ is valid. Furthermore, it follows easily that

$$
\beta_{n}\left\langle\Psi_{n}(z), z^{n}\right\rangle=\gamma_{n}\left\langle\Psi_{n-1}(z), z^{n-1}\right\rangle .
$$

Hence,

$$
\left\langle\Psi_{n}(z), z^{n}\right\rangle=c_{0} \prod_{k=1}^{n} \frac{\gamma_{k}}{\beta_{k}}=: b_{n} \neq 0 .
$$

If a sequence of POPUC associated with $\mu$ exists, it is uniquely determined by $b_{0}$ and $b_{n}$. Therefore,

$$
\left\langle\Psi_{n}, 1\right\rangle=b_{0}, \quad\left\langle\Psi_{n}, z^{k}\right\rangle=0, \quad 1 \leq k \leq n-1, \quad\left\langle\Psi_{n}, z^{n}\right\rangle=b_{n},
$$

is a consistent system of $n+1$ linear equations with $n+1$ unknowns. Notice that the coefficient matrix of this system is the moment matrix $\boldsymbol{T}_{n}$. Then it has a unique solution determined by $b_{0}$ and $b_{n}$ so that $\operatorname{det}\left(\boldsymbol{T}_{n}\right) \neq 0$. Thus, there exists a 
quasidefinite moment functional $\mu$ such that $\left\{\Psi_{n}\right\}_{n \geq 0}$ is the corresponding sequence of POPUC. This proves the first part of the theorem.

Let us now assume that $\left\{\Psi_{n}\right\}_{n \geq 0}$ is a sequence of polynomials with all its zeros on $\partial \mathbb{D}$. By Cohn's theorem [Rahman and Schmeisser 2002], we know that a polynomial with all its zeros on $\partial \mathbb{D}$ must be invariant. Furthermore, by Chen's theorem [1995, Theorem 1], we also know that a necessary and sufficient condition for all the zeros of $\Psi_{n}$ to lie on $\partial \mathbb{D}$ is that there exists a polynomial $\pi_{n-l} \in$ $\mathbb{P}_{n-l} \backslash \mathbb{P}_{n-l-1}$ with all its zeros in $\mathbb{D}$ or on $\partial \mathbb{D}$ such that

$$
\Psi_{n}(z)=z^{l} \pi_{n-l}(z)-\zeta_{n} \pi_{n-l}^{*}(z)
$$

for some nonnegative integer $l$ and $\zeta_{n} \in \partial \mathbb{D}$. By the first part of the theorem, $\left\{\Psi_{n}\right\}_{n \geq 0}$ is a sequence of POPUC with respect to a quasidefinite functional $\mu$. By [Jones et al. 1989, Theorem 6.1(B)], (3-18) holds for $l \equiv 1, \zeta_{n}=\bar{\tau}_{n}$ and $\pi_{n-1}=\Phi_{n-1}$. We recall that $\left\{\Phi_{n}\right\}_{n \geq 0}$ is the sequence of OPUC associated with the POPUC $\left\{\Psi_{n}\right\}_{n \geq 0}$ and the functional $\mu$. At this point, we only can guarantee that the zeros of $\Phi_{n-1}$ lie on $\mathbb{C} \backslash \partial \mathbb{D}$ [Marcellán and Godoy 1991, Proposition 3.1]. If the zeros of $\Psi_{n}$ lie on $\partial \mathbb{D}$, then by Chen's theorem the zeros of $\Phi_{n-1}$ lie in $\mathbb{D}$. Finally, by Geronimus' theorem [1946, Theorem I], under our hypothesis the functional $\mu$ is positive definite.

The uniqueness of $\mu$ is a consequence inherited from the associated OPUC.

It is very well known that for any three contiguous hypergeometric functions there is a linear contiguous relation. So if we are looking for a sequence of polynomials satisfying (3-7), we can find examples if we consider hypergeometric polynomials [Andrews et al. 1999, (2.5.16)]. Notice that from the previous theorem,

$$
\begin{aligned}
& (c+n)_{2} F_{1}(-n-1, b ; c ; 1-z) \\
& \quad=((b+n) z+c-b+n)_{2} F_{1}(-n, b ; c ; 1-z)-n z_{2} F_{1}(-n+1, b ; c ; 1-z),
\end{aligned}
$$

where $b, c \in \mathbb{C}$ and $c \neq 0,-1,-2, \ldots$ gives a set of hypergeometric POPUC.

Example 3.5 (Askey POPUC). An example of polynomials satisfying (3-10) are the hypergeometric polynomials

$$
\frac{(2 a)_{n}}{(a+b i)_{n}}{ }_{2} F_{1}(-n, a+b i ; 2 a ; 1-z), \quad a>0, \quad b \in \mathbb{R}, \quad n \geq 1,
$$

where $(a)_{n}$ denotes the Pochhammer symbol defined by $(a)_{0}:=1$ and $(a)_{n}:=$ $a(a+1) \cdots(a+n-1)$, and the recurrence coefficients are particularly chosen as

$$
\beta_{n}=\frac{a+n-b i}{a+n+b i} \in \partial \mathbb{D}, \quad \gamma_{n}=\frac{n(2 a+n-1)}{(a+b i+n-1)(a+b i+n)} \in \mathbb{C} \backslash\{0\} .
$$

It is easy to check that the polynomials (3-20) have all their zeros on $\partial \mathbb{D}$. Thus, in accordance with Theorem 3.4, there exists a unique nontrivial probability measure $d \sigma$ supported on $\partial \mathbb{D}$ such that the polynomials (3-20) are the corresponding POPUC. 
These polynomials are a particular case of the so-called Askey POPUC; see [Castillo 2015; Castillo et al. 2014; Dimitrov and Sri Ranga 2013].

Now we can go a step further with respect to the above example in order to obtain of some known results as a direct consequence of our study.

Example 3.6 (Delsarte-Genin POPUC). Let $\left\{b_{n}\right\}_{n \geq 0}$ be an arbitrary sequence of nonzero real numbers, let $\left\{a_{n}\right\}_{n \geq 1}$ be an arbitrary positive chain sequence [Chihara 1978], and let $\left\{\varphi_{n}\right\}_{n \geq 0}$ be the sequence of polynomials recursively defined by

$$
\varphi_{n+1}(z)=\left(\left(1+i b_{n}\right) z+\left(1-i b_{n}\right)\right) \varphi_{n}(z)-4 a_{n} z \varphi_{n-1}(z),
$$

with initial conditions $\varphi_{0}:=1$ and $\varphi_{1}(z):=\left(1+i b_{0}\right) z+\left(1-i b_{0}\right)$. It is worth mentioning that (3-22) is the recurrence relation studied by Delsarte and Genin [1988; 1991a; 1991b; 1990], among others. The interlacing property on $\partial \mathbb{D}$ of the zeros of $\left\{\varphi_{n}\right\}_{n \geq 0}$ was recently proved in [Dimitrov and Sri Ranga 2013, Theorem 1.1]; see also [Castillo et al. 2014], although it was first proved in [Delsarte and Genin 1988, Section 5]. In any case, an easy computation shows that these polynomials satisfy the conditions of Theorem 3.4 with all their zeros on $\partial \mathbb{D}$. So, the interlacing property of the zeros of $\left\{\varphi_{n}\right\}_{n \geq 0}$ is also a direct consequence of the fact that from Theorem 3.4, these polynomials are POPUC associated with some nontrivial probability measure $d \sigma$ supported on $\partial \mathbb{D}$. Actually, we can say much more about the behavior of their zeros using the known results for the zeros of POPUC; see, e.g., [Cantero et al. 2002; Golinskii 2002; Simon 2005a; 2005b; 2007; 2011; Wong 2007].

An interesting and nontrivial extension of the results of this section is the connection with those obtained in [Lamblém et al. 2010] where a non-Hermitian linear functional $\tilde{\mu}$ on $\Lambda$ satisfying $c_{n}=c_{-n} \in \mathbb{C}$ is considered (compare with (2-1)) yielding the definition of Szegö-type polynomials. We recall that the case $c_{n}=c_{-n} \in \mathbb{R}$ was previously considered in [Delsarte and Genin 1986].

\section{Applications}

The aim of this last section is to establish two applications of the results presented in the previous section.

Analytic theory of polynomials. The major role of POPUC, as it was pointed out in the Introduction is played by the zero behavior. The results in this direction can be divided into two sets, depending on the methodology used by the authors. The first one is composed by Cantero, Moral and Velázquez [Cantero et al. 2002], Golinskii [2002], and Wong [2007], whose basic tool is the Christoffel-Darboux formula. The second one is by Simon [2007] who used the theory of rank-one perturbations of unitary matrices. 
Although many of the results of zeros of POPUC are well known, a natural question is still open: are two polynomials with simple and strictly interlacing zeros on $\partial \mathbb{D}$, with the possible exception of one of them which could be common, elements of a sequence of POPUC? After the previous section it is natural to conjecture that the answer will be yes. The following result for OPRL goes back to the work of Wendroff [1961]. We must emphasize that it was known by Geronimus [1946, pp. 744] also for OPUC.

Theorem 4.1 (Geronimus-Wendroff theorem). Let $\Psi_{n}$ and $\Psi_{n+1}$ be two monic polynomials whose zeros are simple and strictly interlacing on $\partial \mathbb{D}$. Then there exists a measure $d \sigma$ for which they are POPUC. All such measures have the same $\Psi_{j}$, $0 \leq j \leq n+1$. Moreover, if $\Psi_{n}$ and $\Psi_{n+1}$ have at most one zero in common, the statement of the theorem is also true.

Proof. Let us assume that the zeros of $\Psi_{n}$ and $\Psi_{n+1}$ are strictly interlacing on $\partial \mathbb{D}$. The same idea can be used for the case that $\Psi_{n}$ and $\Psi_{n+1}$ have one zero in common. Let $\left\{e^{i \theta_{n, k}}\right\}_{k=1}^{n}$ be the zeros of $\Psi_{n}$. Set

$$
\beta_{n}:=-e^{i \sum_{k=1}^{n+1} \theta_{n+1, k}} e^{-i \sum_{k=1}^{n} \theta_{n, k}} \in \partial \mathbb{D} .
$$

Notice that the polynomial

$$
\prod_{k=1}^{n+1}\left(z-e^{i \theta_{n+1, k}}\right)-\left(z+\beta_{n}\right) \prod_{k=1}^{n}\left(z-e^{i \theta_{n, k}}\right)
$$

has a zero at $z=0$. Then,

$$
\Psi_{n+1}(z)-\left(z+\beta_{n}\right) \Psi_{n}(z)=-\gamma_{n} z B_{r}(z),
$$

where $B_{r}$ is a monic polynomial of degree at most $n-1$. Since $e^{i \theta_{n+1, k}}-\beta_{n} \neq 0$ and $\Psi_{n}\left(e^{i \theta_{n+1, k}}\right) \neq 0$, we have that $\gamma_{n} \neq 0$ and $B_{r}\left(e^{i \theta_{n+1, k}}\right) \neq 0$. Furthermore,

$$
\Psi_{n+1}\left(e^{i \theta_{n, k}}\right)=-\gamma_{n} e^{i \theta_{n, k}} B_{r}\left(e^{i \theta_{n, k}}\right) .
$$

It is known that an arbitrary polynomial with simple zeros on $\partial \mathbb{D}$ is a POPUC with respect to some nontrivial probability measure supported on $\partial \mathbb{D}$ [Castillo et al. 2015]. Since $\Psi_{n}\left(\beta_{n}\right) \neq 0$ and $\Psi_{n+1}\left(\beta_{n}\right) \neq 0$, and we are interested in the zeros, there is no loss of generality if we assume that

$$
\begin{aligned}
\left(z+\beta_{n}\right) \Psi_{n}(z) & =\bar{\beta} P_{n+1}(z)-\beta P_{n+1}^{*}(z), & & \beta \in \mathbb{C} \backslash\{0\}, \\
\Psi_{n+1}(z) & =\bar{\alpha} Q_{n+1}(z)-\alpha Q_{n+1}^{*}(z), & & \alpha \in \mathbb{C} \backslash\{0\},
\end{aligned}
$$

where $P_{n+1}(z)$ and $Q_{n+1}(z)$ are the OPUC associated with $\left(z+\beta_{n}\right) \Psi_{n}(z)$ and $\Psi_{n+1}(z)$, respectively. Hence,

$$
-\gamma_{n} z B_{r}(z)=\left(\bar{\alpha} P_{n+1}(z)-\bar{\beta} Q_{n+1}(z)\right)+\left(\alpha P_{n+1}^{*}(z)-\beta Q_{n+1}^{*}(z)\right) .
$$


Let us introduce two auxiliary functions

$$
f_{n}(\theta):=\frac{\Psi_{n}(z)}{i z^{n / 2}}, \quad g_{r}(\theta):=\frac{z B_{r}(z)}{i z^{(n+1) / 2}},
$$

where $\left(r e^{i \theta}\right)^{1 / 2}=\sqrt{r} e^{i \theta / 2}, r>0$, and $\theta \in(\tilde{\omega}, \tilde{\omega}+2 \pi)$. Clearly, $f_{n}(\theta)$ and $g_{r}(\theta)$ are real-valued $C^{\infty}$ functions defined on $(\tilde{\omega}, \tilde{\omega}+2 \pi)$ and, by definition they have the same number of zeros on $(\tilde{\omega}, \tilde{\omega}+2 \pi)$ as $\Psi_{n}$ and $B_{r}$ on $\partial \mathbb{D}$, respectively.

One denotes the zeros of $f_{n}$ by $x_{n, k}, k=1, \ldots, n$. As a consequence of the interlacing hypothesis, we have

$$
f_{n+1}\left(x_{n, k+1}\right) f_{n+1}\left(x_{n, k}\right)<0 .
$$

Therefore,

$$
f_{n+1}\left(x_{n, k+1}\right) f_{n+1}\left(x_{n, k}\right)=\left|\gamma_{n}\right|^{2} g_{r}\left(x_{n, k}\right) g_{r}\left(x_{n, k+1}\right),
$$

from which

$$
g_{r}\left(x_{n, k}\right) g_{r}\left(x_{n, k+1}\right)<0 .
$$

This implies that $B_{r}$ has $n-1$ zeros strictly interlacing on $\partial \mathbb{D}$ with the zeros of $\Psi_{n}$. If we define the polynomial $\Psi_{n-1}: \equiv B_{r}$, we can construct, just repeating the above procedure, a polynomial of degree $n-2$ whose zeros interlace with those of $\Psi_{n-1}$. So we can find all the polynomials (uniquely determined) with degree less than $n-1$. By the above construction, the polynomials $\Psi_{j}, 0 \leq j \leq n-1$, are determined by $\Psi_{n}$ and $\Psi_{n+1}$. Finally, applying Theorem 3.4, the result is proved.

Computation of Szegó quadrature formulas. In some applications and theoretical problems, it is of interest to compute $\Phi_{n}\left(\cdot, \tau_{n}\right)$ for some $n \geq 1$ and a fixed $\tau_{n} \in \partial \mathbb{D}$. A motivation to this problem can be given when the estimation of the integral

$$
I_{\sigma}(f):=\int f(z) d \sigma(z)
$$

is considered by means of SQ formulas,

$$
I_{n}(f):=\sum_{j=1}^{n} \lambda_{j} f\left(z_{j}\right), \quad z_{j} \in \partial \mathbb{D}, \quad j=1, \ldots, n, \quad z_{j} \neq z_{k} \text { if } j \neq k, \quad n \geq 1 .
$$

Here, the nodes $\left\{z_{j}\right\}_{j=1}^{n}$ and weights $\left\{\lambda_{j}\right\}_{j=1}^{n}$ are determined so that $I_{n}(f)=I_{\sigma}(f)$ for all functions $f$ belonging to a subspace of $\Lambda$ whose dimension is as large as possible. The "optimal" subspace of exactness is $\Lambda_{-(n-1), n-1}$ (of dimension $2 n-1$ ), and this one-parameter family of optimal SQ formulas can be characterized as:

(i) The nodes are the zeros of an $n$-th POPUC associated with the measure $d \sigma$.

(ii) The weights can be computed by

$$
\lambda_{j}=-\frac{1}{2 z_{j}} \frac{\Upsilon_{n}\left(z_{j}, \tau_{n}\right)}{\Phi_{n}^{\prime}\left(z_{j}, \tau_{n}\right)}>0, \quad j=1, \ldots, n,
$$


where $\Upsilon_{n}\left(\cdot, \tau_{n}\right)$ represents the corresponding $n$-th second kind POPUC, which can be obtained from (2-6) with the same $\tau_{n}$ and Verblunsky coefficients $\left\{-\alpha_{n}\right\}_{n \geq 0}$ (see [Wong 2007]).

The positive character of the weights is of importance for stability and convergence reasons. If you fix one or two nodes in advance in (4-24) then you get an extension of the classical Gauss-Radau and Gauss-Lobatto quadrature formulas for measures supported on the real line. But the situation on the unit circle is completely different. Because of the dependence of the parameter $\tau_{n}$, Szegó-Radau quadrature formulas can be always constructed by taking an appropriate selection of the parameter $\tau_{n}$ : if we want $\zeta \in \partial \mathbb{D}$ to be a node of the rule, then

$$
\Phi_{n}\left(\zeta, \tau_{n}\right)=0 \quad \Longleftrightarrow \quad \zeta \Phi_{n-1}(\zeta)-\bar{\tau}_{n} \Phi_{n-1}^{*}(\zeta)=0 \quad \Longleftrightarrow \quad \tau_{n}=\zeta^{n-2} \frac{\Phi_{n-1}(\zeta)}{\overline{\Phi_{n-1}(\zeta)}},
$$

and from Heine's formula [Simon 2005a, Theorem 1.5.11(a)], it is expressed in terms of $\zeta$ and the trigonometric moments of the measure $d \sigma$ :

$$
\tau_{n}=\zeta^{n-2} \frac{\Delta}{\bar{\Delta}}, \quad \Delta:=\operatorname{det}\left[\begin{array}{cccc}
c_{0} & c_{1} & \cdots & c_{n-1} \\
c_{-1} & c_{0} & \cdots & c_{n-2} \\
\vdots & \vdots & & \vdots \\
c_{-n+2} & c_{-n+3} & \cdots & c_{1} \\
1 & \zeta & \cdots & \zeta^{n-1}
\end{array}\right] .
$$

The construction of Szegó-Lobatto quadrature formulas also requires the computation of an $n$-th POPUC with a specific value of the parameter $\tau_{n}$; see [Cruz-Barroso et al. 2015].

Another motivation to the same problem, concerning the construction of interpolatory quadrature formulas for the estimation of integrals with respect to measures supported on intervals of the real line can be found in [Bultheel et al. 2005]. In that paper, $n$-point positive interpolatory quadrature formulas on $[-1,1]$ are constructed by taking as nodes the real part of some of the zeros of certain POPUC, and it is also proved there that an appropriate selection of the paraorthogonality parameter makes it always possible to obtain "optimal" rules, exact in a subspace of algebraic polynomials of dimension $n+1$.

The results of Section 3 can be used to solve this problem in an alternative way, by computing directly a sequence of POPUC, instead of the associated OPUC. Indeed, let us consider first the initial conditions of the three-term recurrence for POPUC. For arbitrary $\tau_{1} \in \partial \mathbb{D}$, set

$$
\Phi_{-1}\left(\cdot, \tau_{-1}\right): \equiv 0, \quad \Phi_{0}\left(\cdot, \tau_{0}\right): \equiv 1, \quad \Phi_{1}\left(z, \tau_{1}\right)=z-\bar{\tau}_{1},
$$

so if we define $\tau_{0}:=-1$, the formula for $\beta_{n}$ in Corollary 3.2 holds for $n=0$. Set now $\tau_{2} \in \partial \mathbb{D}$ also arbitrary, and $\beta_{1}=\tau_{1} \bar{\tau}_{2}$. From the combination of Theorem 3.1 
and (3-11) with $n=1$, and (2-6) with $n=2$, it follows that

$$
\Phi_{2}\left(z, \tau_{2}\right)=z^{2}+\left(\bar{\tau}_{2} \alpha_{0}-\bar{\alpha}_{0}\right) z-\bar{\tau}_{2}, \quad \gamma_{1}=\bar{\tau}_{2}\left(\tau_{1}-\alpha_{0}\right)-\left(\bar{\tau}_{1}-\bar{\alpha}_{0}\right) .
$$

So, with these initial conditions, we are able to use (3-7) for the computation of a particular sequence of POPUC that depends on the free selection of $\tau_{1}, \tau_{2} \in \partial \mathbb{D}$ : for $n \geq 2$, we compute $\tau_{n+1}$ from (3-13), and hence $\beta_{n}$ and $\gamma_{n}$ are obtained from Corollary 3.2. Now, the key fact is that since the recurrence relation for the paraorthogonality parameters is invertible (see (3-14)), and both depend only on the measure $d \sigma$, the sequence $\left\{\tau_{k}\right\}_{k=0}^{n+1}$ can be also obtained starting from a fixed $\tau_{n+1}$ and an arbitrary $\tau_{n}$, until we get $\tau_{2}$ (from the initial conditions, $\tau_{1}$ will not be needed and notice that $\alpha_{-1}:=-1$ always implies $\tau_{0}=-1$ ). The remaining parameters $\beta_{n}$ and $\gamma_{n}$ in the recurrence are thus directly obtained from Corollary 3.2 for $n \geq 2$.

To end, let us illustrate the method. Despite what happens to OPRL, few measures on the unit circle provide families of POPUC that are explicitly given. A known family of measures of importance is the Jacobi-type weight functions

$$
d \sigma_{\alpha, \beta}(\theta)=(1-\cos \theta)^{\alpha+1 / 2}(1+\cos \theta)^{\beta+1 / 2} d \theta, \quad \alpha, \beta>-1, \quad \theta \in[0,2 \pi),
$$

with Verblunsky coefficients

$$
\alpha_{n}=\frac{(-1)^{n}\left(\beta+\frac{1}{2}\right)-\alpha-\frac{1}{2}}{n+\alpha+\beta+2},
$$

but for which only for the four Chebyshev-type weight functions $\alpha, \beta \in\{ \pm 1 / 2\}$ are there explicit expressions for POPUC (see [Daruis et al. 2002]). For other selections of $\alpha, \beta$, we can compute $\Phi_{n}\left(\cdot, \tau_{n}\right)$ for $d \sigma_{\alpha, \beta}, n \geq 2$, and a prescribed $\tau_{n}$ from our three-term recurrence. An example is given below.

Example 4.2. Notice that since $d \sigma_{\alpha, \beta}$ is a symmetric weight $\left(\alpha_{n} \in \mathbb{R}\right)$, the polynomial $\Phi_{n}(\cdot, \pm 1)$ will have real coefficients, and the nonreal zeros will appear in complex conjugate pairs.

(i) Setting $\alpha=0, \beta=1$ and $\tau_{1}=\tau_{2}=1$, we obtain from the forward recurrence (3-13) that $\tau_{15}=1$ and that $\Phi_{15}(z, 1)=\sum_{j=0}^{7} a_{j}\left(z^{j}-z^{15-j}\right)$ is given by $a_{0}=-a_{1}=-1, a_{2}=-a_{3}=-7 / 5, a_{4}=-a_{5}=-21 / 13$ and $a_{6}=-a_{7}=$ $-245 / 143$.

(ii) Set $\alpha=\beta=1 / 4$ :

(a) If $\tau_{10}=1$ and $\tau_{9}=i$, we can make use of the backward recurrence (3-14) to obtain $\tau_{2}=1$ and $\tau_{1}=i$.

(b) For the choice $\tau_{14}=(\sqrt{2} / 2)(1+i)$, the corresponding POPUC and second kind POPUC have been computed from the three-term recurrence. The nodes and weights (obtained from (4-25)) of the 14-point SQ formula are displayed in Table 1. 


\begin{tabular}{|rc|}
\hline \multicolumn{1}{|c|}{ nodes } & weights \\
\hline $0.953562637438023+0.405929829869375 i$ & 0.010335586974718 \\
$0.719435950626376+0.750717509685024 i$ & 0.022827404435354 \\
$0.366191713059248+0.974873401744596 i$ & 0.032048528041628 \\
$-0.047525423154063+1.041505278940548 i$ & 0.034116841058949 \\
$-0.454499431518169+0.939759403074777 i$ & 0.028200751436574 \\
$-0.789255597539979+0.686392494558016 i$ & 0.016744575125821 \\
$-1.000962132835750+0.324853472172218 i$ & 0.005014789370671 \\
$1.000962132835750-0.324853472172218 i$ & 0.005014789370670 \\
$-0.953562637438025-0.405929829869376 i$ & 0.010335586974718 \\
$0.789255597539980-0.686392494558019 i$ & 0.016744575125820 \\
$-0.719435950626374-0.750717509685023 i$ & 0.022827404435355 \\
$0.454499431518170-0.939759403074778 i$ & 0.028200751436573 \\
$-0.366191713059248-0.974873401744598 i$ & 0.032048528041627 \\
$0.047525423154063-1.041505278940550 i$ & 0.034116841058948 \\
\hline
\end{tabular}

Table 1. Nodes and weights of the 14-point SQ formula for $d \sigma_{\alpha, \beta}$ with $\alpha=\beta=1 / 4$, computed from the three-term recurrence relation for the corresponding POPUC and second kind POPUC.

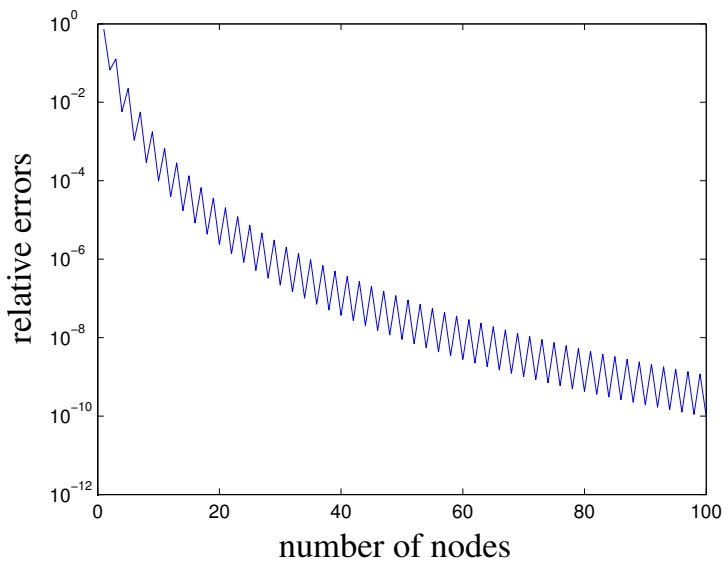

Figure 1. The relative errors in the estimation of $I_{\sigma_{\alpha, \beta}}(f)$ with $\alpha=$ $\beta=1$ and $f(z)=\cos ^{2} \theta$, with $z=e^{i \theta}$, by SQ formulas obtained from the three-term recursion for POPUC and second kind POPUC by taking $\tau_{1}=\tau_{2}=1$.

(iii) Setting $\alpha=\beta=1$, and $f(z)=\cos ^{2} \theta$, with $z=e^{i \theta}$, the relative errors obtained in the estimation of $I_{\sigma_{\alpha, \beta}}(f)$ by using SQ formulas computed via three-term recursion for POPUC and second kind POPUC by taking $\tau_{1}=\tau_{2}=1$ are displayed in Figure 1. 
For numerical reasons, the computation of the zeros of POPUC is preferably done from an eigenvalue problem of certain structured matrices (Hessenberg, CMV, snake-shaped) in a very fast and accurate way. The computations of our method can be arranged so that the nodal polynomial can be determined in only $o(n)$ arithmetic floating point operations. So, it should be said that this alternative procedure is competitive with respect to other procedures already known in the literature, but it is not really an improved algorithm. In any case, our work gives a new perspective to be considered in more detail.

\section{Acknowledgements}

The authors thank the referee for her/his valuable suggestions and comments which have contributed to improve the final form of this paper. The research of the first author is supported by the Portuguese Government through the Fundação para a Ciência e a Tecnologia (FCT) under the grant SFRH/BPD/101139/2014 and partially supported by the Brazilian Government through the CNPq under the project 470019/2013-1 and the Dirección General de Investigación Científica y Técnica, Ministerio de Economía y Competitividad of Spain under the project MTM2012-36732-C03-01. The work of the second and third authors is partially supported by Dirección General de Programas y Transferencia de Conocimiento, Ministerio de Ciencia e Innovación of Spain under the project MTM2011-28781.

\section{References}

[Andrews et al. 1999] G. E. Andrews, R. Askey, and R. Roy, Special functions, Encyclopedia of Mathematics and its Applications 71, Cambridge Univ. Press, 1999. MR 2000g:33001 Zbl 0920.33001

[Atkinson 1964] F. V. Atkinson, Discrete and continuous boundary problems, Mathematics in Science and Engineering 8, Academic Press, New York, 1964. MR 31 \#416 Zbl 0117.05806

[Bultheel et al. 1999] A. Bultheel, P. González-Vera, E. Hendriksen, and O. Njåstad, Orthogonal rational functions, Cambridge Monographs on Applied and Computational Mathematics 5, Cambridge Univ. Press, 1999. MR 2000c:33001 Zbl 0923.42017

[Bultheel et al. 2005] A. Bultheel, L. Daruis, and P. González-Vera, "Positive interpolatory quadrature formulas and para-orthogonal polynomials", J. Comput. Appl. Math. 179:1-2 (2005), 97-119. MR 2005m:41061 Zbl 1068.41048

[Cantero et al. 2002] M. J. Cantero, L. Moral, and L. Velázquez, "Measures and para-orthogonal polynomials on the unit circle", East J. Approx. 8:4 (2002), 447-464. MR 2004i:42021 Zbl 05199971

[Castillo 2015] K. Castillo, "Monotonicity of zeros for a class of polynomials including hypergeometric polynomials", Appl. Math. Comput. 266 (2015), 183-193. MR 3377550

[Castillo et al. 2014] K. Castillo, M. S. Costa, A. Sri Ranga, and D. O. Veronese, "A Favard type theorem for orthogonal polynomials on the unit circle from a three term recurrence formula", $J$. Approx. Theory 184 (2014), 146-162. MR 3218796 Zbl 1291.42021

[Castillo et al. 2015] K. Castillo, F. Marcellán, and M. N. Rebocho, "Zeros of para-orthogonal polynomials and linear spectral transformations on the unit circle", Numer. Algorithms (online publication June 2015). 
[Chen 1995] W. Chen, "On the polynomials with all their zeros on the unit circle", J. Math. Anal. Appl. 190:3 (1995), 714-724. MR 96b:30011 Zbl 0822.30008

[Chihara 1978] T. S. Chihara, An introduction to orthogonal polynomials, Mathematics and its Applications 13, Gordon and Breach, New York, 1978. MR 58 \#1979 Zbl 0389.33008

[Cruz-Barroso et al. 2007] R. Cruz-Barroso, L. Daruis, P. González-Vera, and O. Njåstad, "Sequences of orthogonal Laurent polynomials, bi-orthogonality and quadrature formulas on the unit circle", $J$. Comput. Appl. Math. 200:1 (2007), 424-440. MR 2007j:65032 Zbl 1136.42020

[Cruz-Barroso et al. 2015] R. Cruz-Barroso, C. Díaz Mendoza, and F. Perdomo-Pío, "A connection between Szegő-Lobatto and quasi Gauss-type quadrature formulas", J. Comput. Appl. Math. 284 (2015), 133-143. MR 3319499 Zbl 1314.65039

[Daruis et al. 2002] L. Daruis, P. González-Vera, and O. Njåstad, "Szegö quadrature formulas for certain Jacobi-type weight functions”, Math. Comp. 71:238 (2002), 683-701. MR 2002k:41043 Zbl 0989.41015

[Daruis et al. 2003] L. Daruis, O. Njåstad, and W. Van Assche, "Para-orthogonal polynomials in frequency analysis", Rocky Mountain J. Math. 33:2 (2003), 629-645. MR 2004j:42019 Zbl 1042.94001

[Delsarte and Genin 1986] P. Delsarte and Y. V. Genin, “The split Levinson algorithm”, IEEE Trans. Acoust. Speech Signal Process. 34:3 (1986), 470-478. MR 87f:94007

[Delsarte and Genin 1988] P. Delsarte and Y. Genin, “The tridiagonal approach to Szegő's orthogonal polynomials, Toeplitz linear systems, and related interpolation problems", SIAM J. Math. Anal. 19:3 (1988), 718-735. MR 89d:30040 Zbl 0638.30037

[Delsarte and Genin 1990] P. Delsarte and Y. Genin, "On the role of orthogonal polynomials on the unit circle in digital signal processing applications", pp. 115-133 in Orthogonal polynomials (Columbus, OH, 1989), edited by P. Nevai, NATO Adv. Sci. Inst. Ser. C Math. Phys. Sci. 294, Kluwer Acad. Publ., Dordrecht, 1990. MR 92b:94002 Zbl 0704.42026

[Delsarte and Genin 1991a] P. Delsarte and Y. Genin, "Tridiagonal approach to the algebraic environment of Toeplitz matrices, I: Basic results", SIAM J. Matrix Anal. Appl. 12:2 (1991), 220-238. MR 92d:65071 Zbl 0728.65020

[Delsarte and Genin 1991b] P. Delsarte and Y. Genin, "Tridiagonal approach to the algebraic environment of Toeplitz matrices, II: Zero and eigenvalue problems", SIAM J. Matrix Anal. Appl. 12:3 (1991), 432-448. MR 92d:65072 Zbl 0753.15015

[Delsarte et al. 1978] P. Delsarte, Y. V. Genin, and Y. G. Kamp, "Orthogonal polynomial matrices on the unit circle", IEEE Trans. Circuits and Systems CAS-25:3 (1978), 149-160. MR 58 \#1981 Zbl 0408.15018

[Dimitrov and Sri Ranga 2013] D. K. Dimitrov and A. Sri Ranga, "Zeros of a family of hypergeometric para-orthogonal polynomials on the unit circle", Math. Nachr. 286:17-18 (2013), 1778-1791. MR 3145170 Zbl 1290.33008

[Erdélyi et al. 1991] T. Erdélyi, P. Nevai, J. Zhang, and J. S. Geronimo, "A simple proof of "Favard's theorem" on the unit circle", Atti Sem. Mat. Fis. Univ. Modena 39:2 (1991), 551-556. MR 93a:42010 Zbl 0753.33004

[Favard 1935] J. Favard, "Sur les polynômes de Tchebycheff”, C. R. Acad. Sci. Paris 200 (1935), 2052-2055. Zbl 0012.06205

[Geronimus 1946] J. Geronimus, "On the trigonometric moment problem", Ann. of Math. (2) 47 (1946), 742-761. MR 8,265d Zbl 0060.16903

[Golinskii 2002] L. Golinskii, "Quadrature formula and zeros of para-orthogonal polynomials on the unit circle”, Acta Math. Hungar. 96:3 (2002), 169-186. MR 2003e:41048 Zbl 1017.42014

[Helsen et al. 2005] S. Helsen, A. B. J. Kuijlaars, and M. Van Barel, "Convergence of the isometric Arnoldi process", SIAM J. Matrix Anal. Appl. 26:3 (2005), 782-809. MR 2006d:65037 Zbl 1082.65038 
[Ismail 2005] M. E. H. Ismail, Classical and quantum orthogonal polynomials in one variable, Encyclopedia of Mathematics and its Applications 98, Cambridge Univ. Press, 2005. MR 2007f:33001 Zbl 1082.42016

[Jones et al. 1989] W. B. Jones, O. Njåstad, and W. J. Thron, "Moment theory, orthogonal polynomials, quadrature, and continued fractions associated with the unit circle", Bull. London Math. Soc. 21:2 (1989), 113-152. MR 90e:42027 Zbl 0637.30035

[Lamblém et al. 2010] R. L. Lamblém, J. H. McCabe, M. A. Piñar, and A. Sri Ranga, "Szegő type polynomials and para-orthogonal polynomials", J. Math. Anal. Appl. 370:1 (2010), 30-41. MR 2011g:42066 Zbl 1213.42092

[Marcellán and Álvarez-Nodarse 2001] F. Marcellán and R. Álvarez-Nodarse, "On the "Favard theorem" and its extensions", J. Comput. Appl. Math. 127:1-2 (2001), 231-254. MR 2001m:42048 Zbl 0970.33008

[Marcellán and Godoy 1991] F. Marcellán and E. Godoy, "Orthogonal polynomials on the unit circle: Distribution of zeros", J. Comput. Appl. Math. 37:1-3 (1991), 195-208. MR 92m:42027 Zbl 0745.42013

[Peherstorfer 2011] F. Peherstorfer, "Positive trigonometric quadrature formulas and quadrature on the unit circle", Math. Comp. 80:275 (2011), 1685-1701. MR 2012d:65051 Zbl 1221.42003

[Rahman and Schmeisser 2002] Q. I. Rahman and G. Schmeisser, Analytic theory of polynomials, London Mathematical Society Monographs. New Series 26, Clarendon Press, Oxford, 2002. MR 2004b:30015 Zbl 1072.30006

[Simanek 2015] B. Simanek, "An electrostatic interpretation of the zeros of paraorthogonal polynomials on the unit circle", preprint, 2015. arXiv 1501.05672

[Simon 2005a] B. Simon, Orthogonal polynomials on the unit circle, I: Classical theory, American Mathematical Society Colloquium Publications 54, Amer. Math. Soc., Providence, RI, 2005. MR 2006a:42002a Zbl 1082.42020

[Simon 2005b] B. Simon, Orthogonal polynomials on the unit circle, II: Spectral theory, American Mathematical Society Colloquium Publications 54, Amer. Math. Soc., Providence, RI, 2005. MR 2006a:42002b Zbl 1082.42021

[Simon 2007] B. Simon, "Rank one perturbations and the zeros of paraorthogonal polynomials on the unit circle", J. Math. Anal. Appl. 329:1 (2007), 376-382. MR 2008c:42027 Zbl 1110.33004

[Simon 2011] B. Simon, Szegó's theorem and its descendants: Spectral theory for $L^{2}$ perturbations of orthogonal polynomials, Princeton Univ. Press, 2011. MR 2012b:47080 Zbl 1230.33001

[Stieltjes 1894] T.-J. Stieltjes, "Recherches sur les fractions continues", Ann. Fac. Sci. Toulouse Sci. Math. Sci. Phys. 8:4 (1894), J1-J122. MR 1508159 JFM 25.0326.01

[Stieltjes 1895] T.-J. Stieltjes, "Recherches sur les fractions continues [Suite et fin]", Ann. Fac. Sci. Toulouse Sci. Math. Sci. Phys. 9:1 (1895), A5-A47. MR 1508160 JFM 25.0326.01

[Stone 1932] M. H. Stone, Linear transformations in Hilbert space, American Mathematical Society Colloquium Publications 15, Amer. Math. Soc., Providence, RI, 1932. Zbl 0005.40003

[Szegó 1975] G. Szegó, Orthogonal polynomials, 4th ed., American Mathematical Society Colloquium Publications 23, Amer. Math. Soc., Providence, RI, 1975. MR 51 \#8724 Zbl 0305.42011

[Wendroff 1961] B. Wendroff, "On orthogonal polynomials”, Proc. Amer. Math. Soc. 12 (1961), 554-555. MR 24 \#A974 Zbl 0099.05601

[Wong 2007] M. L. Wong, "First and second kind paraorthogonal polynomials and their zeros", $J$. Approx. Theory 146:2 (2007), 282-293. MR 2008f:42028 Zbl 1116.33012

Received February 9, 2015. Revised July 3, 2015. 
Kenier Castillo

CMUC, DePartment of Mathematics

UNIVERSITY OF COIMBRA

3001-501 COIMBRA

PORTUGAL

kcastill@math.uc3m.3s

kenier@mat.uc.pt

RUYMÁN CRUZ-BARROSO

DEPARTAMENTO DE ANÁlisis MATEMÁtico

UNIVERSIDAD DE LA LAGUNA

38271 LA LAGUNA

TENERIFE, CANARY ISLANDS

SPAIN

rcruzb@ull.es

FRANCISCO PERDOMO-PÍO

Departamento de AnÁlisis Matemático

UNIVERSIDAD DE LA LAGUNA

38271 LA LAGUNA

TENERIFE, CANARY ISLANDS

SPAIN

fjppio@ull.es 


\title{
PACIFIC JOURNAL OF MATHEMATICS
}

\author{
msp.org/pjm
}

Founded in 1951 by E. F. Beckenbach (1906-1982) and F. Wolf (1904-1989)

\section{EDITORS}

Don Blasius (Managing Editor)

Department of Mathematics

University of California

Los Angeles, CA 90095-1555

blasius@math.ucla.edu

\author{
Paul Balmer \\ Department of Mathematics \\ University of California \\ Los Angeles, CA 90095-1555 \\ balmer@math.ucla.edu \\ Robert Finn \\ Department of Mathematics \\ Stanford University \\ Stanford, CA 94305-2125 \\ finn@math.stanford.edu \\ Sorin Popa \\ Department of Mathematics \\ University of California \\ Los Angeles, CA 90095-1555 \\ popa@math.ucla.edu
}

\author{
Vyjayanthi Chari \\ Department of Mathematics \\ University of California \\ Riverside, CA 92521-0135 \\ chari@math.ucr.edu \\ Kefeng Liu \\ Department of Mathematics \\ University of California \\ Los Angeles, CA 90095-1555 \\ liu@math.ucla.edu \\ Jie Qing \\ Department of Mathematics \\ University of California \\ Santa Cruz, CA 95064 \\ qing@ cats.ucsc.edu
}

\section{PRODUCTION}

Silvio Levy, Scientific Editor, production@msp.org

\section{SUPPORTING INSTITUTIONS}

ACADEMIA SINICA, TAIPEI

CALIFORNIA INST. OF TECHNOLOGY

INST. DE MATEMÁTICA PURA E APLICADA

KEIO UNIVERSITY

MATH. SCIENCES RESEARCH INSTITUTE

NEW MEXICO STATE UNIV.

OREGON STATE UNIV.

\author{
STANFORD UNIVERSITY \\ UNIV. OF BRITISH COLUMBIA \\ UNIV. OF CALIFORNIA, BERKELEY \\ UNIV. OF CALIFORNIA, DAVIS \\ UNIV. OF CALIFORNIA, LOS ANGELES \\ UNIV. OF CALIFORNIA, RIVERSIDE \\ UNIV. OF CALIFORNIA, SAN DIEGO \\ UNIV. OF CALIF., SANTA BARBARA
}

\author{
Daryl Cooper \\ Department of Mathematics \\ University of California \\ Santa Barbara, CA 93106-3080 \\ cooper@math.ucsb.edu \\ Jiang-Hua Lu \\ Department of Mathematics \\ The University of Hong Kong \\ Pokfulam Rd., Hong Kong \\ jhlu@maths.hku.hk \\ Paul Yang \\ Department of Mathematics \\ Princeton University \\ Princeton NJ 08544-1000 \\ yang@math.princeton.edu
}

These supporting institutions contribute to the cost of publication of this Journal, but they are not owners or publishers and have no responsibility for its contents or policies.

See inside back cover or msp.org/pjm for submission instructions.

The subscription price for 2016 is US $\$ 440 /$ year for the electronic version, and $\$ 600 /$ year for print and electronic.

Subscriptions, requests for back issues and changes of subscribers address should be sent to Pacific Journal of Mathematics, P.O. Box 4163, Berkeley, CA 94704-0163, U.S.A. The Pacific Journal of Mathematics is indexed by Mathematical Reviews, Zentralblatt MATH, PASCAL CNRS Index, Referativnyi Zhurnal, Current Mathematical Publications and Web of Knowledge (Science Citation Index).

The Pacific Journal of Mathematics (ISSN 0030-8730) at the University of California, c/o Department of Mathematics, 798 Evans Hall \#3840, Berkeley, CA 94720-3840, is published twelve times a year. Periodical rate postage paid at Berkeley, CA 94704, and additional mailing offices. POSTMASTER: send address changes to Pacific Journal of Mathematics, P.O. Box 4163, Berkeley, CA 94704-0163.

PJM peer review and production are managed by EditFLOW ${ }^{\circledR}$ from Mathematical Sciences Publishers.

\section{PUBLISHED BY}

\section{mathematical sciences publishers \\ nonprofit scientific publishing}

http://msp.org/

(C) 2016 Mathematical Sciences Publishers 


\section{PACIFIC JOURNAL OF MATHEMATICS}

Volume $280 \quad$ No. $2 \quad$ February 2016

Topological Molino's theory

JESÚS A. ÁlVAREz LóPEZ and MANUEl F. MoreIRA GALICIA

Equivariant principal bundles and logarithmic connections on toric varieties

INDRANIL BISWAS, ARIJIT DEY and MAINAK PODDAR

On a spectral theorem in paraorthogonality theory

Kenier CASTILlo, RuYmán CRUZ-BARRoso and Francisco

PERDOMO-PÍO

Sigma theory and twisted conjugacy, II: Houghton groups and pure

symmetric automorphism groups

DACIBERG L. GONÇALVES and PARAMESWARAN SANKARAN

The second CR Yamabe invariant

PAK TUNG Ho

No hyperbolic pants for the 4-body problem with strong potential

CONNOR JACKMAN and RICHARD MONTGOMERY

Unions of Lebesgue spaces and $A_{1}$ majorants

Greg Knese, John E. M ${ }^{\mathrm{C}}$ CARThy and Kabe Moen

Complex hyperbolic $(3,3, n)$ triangle groups

JOHN R. PARKER, JiEYAN WANG and BAOHUA XIE

Topological aspects of holomorphic mappings of hyperquadrics from $\mathbb{C}^{2}$ to $\mathbb{C}^{3}$

\section{MICHAEL REITER}

2-Blocks with minimal nonabelian defect groups III

BENJAMIN SAMBALE

Number of singularities of stable maps on surfaces 